\title{
Discovering social behaviour variances of younger and older users through social interaction analysis
}

\section{Darren Quinn*, Liming Chen and Maurice Mulvenna}

School of Computing and Mathematics, University of Ulster, Jordanstown, BT37 0QB, UK

E-mail: quinn-d15@email.ulster.ac.uk

E-mail:1.chen@ulster.ac.uk

E-mail: md.mulvenna@ulster.ac.uk

*Corresponding author

\begin{abstract}
The popularity of social networking has risen considerably in recent years, increasing opportunities for social interaction. As an approach, it has the potential to reduce the burden of social isolation for older users. However, the current state of older user engagement requires investigation. In a study, exploring the possibilities of interaction analysis to undercover user behaviours/characteristics, five aspects of younger and older online engagement were investigated comprising; connectivity, length of engagement, application usage, engagement frequency classification and profile maintenance frequency. Results derived from user generated content, enabled direct comparisons on the engagement levels of both cohorts. Results established interaction analysis as an approach to detect user behaviour(s), observing the degree older users failure to return and maintain activity as significant; however, users who do engage, maintain activity with a broad range of functions. Results quantified the interactions and behaviours of two disparate cohorts, determining their key user characteristics.
\end{abstract}

Keywords: online social networks; interaction analysis; older users; younger users.

Reference to this paper should be made as follows: Quinn, D., Chen, L. and Mulvenna, M. (2013) 'Discovering social behaviour variances of younger and older users through social interaction analysis', Int. J. Web Science, Vol. 2, Nos. 1/2, pp.44-65.

Biographical notes: Darren Quinn is a PhD research student in the School of Computing and Mathematics, University of Ulster, UK. He received his BSc in Computing and Information Systems from the University of Ulster and has over ten years' experience in industry in a range of positions. His research interests include social computing, gerontechnology and human computer interaction.

Liming Chen is a Lecturer at the School of Computing and Mathematics, University of Ulster, UK. He received his BSc and MSc in Computing Engineering from Beijing Institute of Technology, China, and DPhil in Artificial Intelligence from De Montfort University, UK. His current research interests include semantic technologies, knowledge management, intelligent agents, pervasive computing and social computing and their applications in smart homes and intelligent environments. 
Maurice Mulvenna is a Professor of Computer Science in the School of Computing and Mathematics at the University of Ulster, UK and a senior member of both the Institute of Electrical and Electronics Engineers and the Association for Computing Machinery. He is also a Chartered Fellow of the British Computer Society.

\section{Introduction}

In a short space of time, online social networking (OSN) applications such as that of Facebook have deeply impacted modern culture (Kwai and Wagner, 2008; Parameswaran and Whinston, 2007). Their social and economic influence has been widely recognised and is reinforced within the EU impact report, which defined social computing as a phenomenon (Ala-Mutka et al., 2009). Although there are a variety of OSN types such as; social networking services (Facebook, Twitter, LinkedIn, etc.) or social media sharing (YouTube, Flickr, Vimeo, etc.), generically their role can be seen as providing opportunities to increase social interaction. Their popularity was largely driven following the development and emergence of Web 2.0 application features. As a technological advance, it empowered the user as authors, enabling user generated content. In more contemporary times, the new wave of devices such as smart phones and tablet computing, are further encouraging user connectivity and the subsequent generation of user content.

As users engage, a rich history of interactions and their subsequent behaviours is amassed. The digital traces that users create from each online interaction provide a host of research opportunities. Research of OSN is increasingly popular and observed from within a broad range of disciplines such as; sociology, anthropology, medicine and computer science (Chew et al., 2011; Wilson and Peterson, 2002; Greene et al., 2011; Cheung et al., 2011). As a specific area of interest, the interaction patterns of user behaviours require investigation. Studies in this area will help to evaluate how users are connecting, but furthermore, they will establish how connectivity compares at individual and group level. In particular, there is a need for research to try and better understand how disparate cohorts compare; an area which to date has received little attention.

Terms such as 'digital natives' by Prenksy (2001), or the 'net generation' by Tapscott (1998), have been coined to try and characterise the cohort of users who were born approximately between 1980 and 1994. Regardless of description, as a generation they are widely acknowledged for being born into a digital age of; mobile phones, videogames and computers, etc. (Howe and Strauss, 2000; Lenhart et al., 2005). Their adoption of technology resulted in them being accredited with a familiarity with and reliance on ICT' (Bennett et al., 2008). Subsequently, it inevitably meant that it would be those younger users, possessing a greater familiarity who would be early adopters of OSN. However, when considering which cohorts could benefit from OSN technology, a primary candidate would be those who are at the highest risk of social isolation. Given the negative connotations isolation has upon health and wellbeing, as a group older people are identified as being particularly vulnerable (Victor et al., 2005). The issue of isolation for older people is a multi-tiered issue comprised of physical, economic and geographical issues (Grenade and Boldy, 2008; Victor et al., 2009). The challenge of an ageing population's vulnerability to social isolation is a substantial one. However, by creating greater opportunities for social connectivity, OSN may hold significant benefits 
for older users. As a vulnerable group who value their social connections and desire to be in touch (Lindley et al., 2009), the benefits of engaging through this modality may be significant, not least for its potential to reduce the state burden on associated healthcare services.

OSN can allow the older user an opportunity to supplement their real world social interactions and networks, thus creating opportunities to maintain and stimulate connections with friends, family or like-minded groups. As users connect, it allows for the sharing of experiences, events and emotions with networks that are becoming increasingly dispersed (Coyle and Vaughn, 2008). From a supply and demand perspective, it would appear that OSN seems to be a perfect match for older people, given their desire to maintain social connectivity. However, although numerous studies have been carried concerning the barriers of engagement of older user interactions with the web (Sayago and Blat, 2009; Hanson, 2009), to date research has failed to directly compare the interactions and behaviours of older users against the core demographic of younger users.

As rationale for comparing the disparate age cohorts of younger and older users, we aim to firstly assess the interaction levels of social networking's core demographic (i.e., younger users) to determine the current state of the art of social networking's most immersed users (Clickymedia, 2010; Corbett, 2010). Secondly, we aim to provide a direct contrast to the behavioural interactions of those users who are viewed as potentially benefiting from an online social platform (i.e., older users). It is viewed that such networks maybe applied as a form of assistive technology to help alleviate the burden of increasing social isolation. As an investigation into the discrete interactions of users, we view this work as an early exploration into the possibilities of interaction analysis and not as finalised conclusions into the online behaviour of all users. The significant knowledge contributions of this research are made in not just extending traditional measurements approaches of online activity, but many benefits can now be gained within a wide range of domains from the quantification of user engagements in online networks, particularly those of older users where their engagement with such technology is a major concern throughout society Results provide an excellent foundation for future research in order to establish reasoning for such differences, providing a foundation to understand how behaviour in such networks maybe analysed. In particular, our research focus will be to develop new eMetric approaches to determine user behaviours and open up areas of debate for research.

\subsection{Related work}

Research into OSN has predominantly been carried out within the domain social network analysis (SNA), an area which traditionally analysed and visualised these highly complex structures, comprising millions of users. To date studies have addressed a broad range issues such as; community detection (Ferrara, 2012; Chau et al., 2007), measurement and analysis (Mislove et al., 2007; Newman and Leicht, 2007), privacy (Ur and Ganapthy, 2009; Ying and $\mathrm{Wu}, 2009$ ), and relationship strength (Xiang and Rogati, 2009; Au Yeung and Iwata, 2011). In particular, Facebook due to its prominence is receiving significant research attention with works embodying analyses of users (Akcora et al., 2011; Hossmann et al., 2011), motivations for use (Burke et al., 2011; Gomes and Pimentel, 2011) and information disclosure (Chen e al., 2011; Krasnova and Veltri, 2010) as 
examples of contemporary research. However, specifically in regard to the measurement and analysis of OSN user behaviour, it is area which to date has received little attention, largely due to the complexities of privacy and security surrounding individual users. Recently, research into the specific area of interaction analysis has gained momentum. Viewed as an extension of SNA, it aims to understand the individual and their use of functions, discovering behavioural patterns and specific user characteristics through a micro level approach. Wilson et al. (2009) posed the question "Are social links valid indicators of real user interaction? If not, then how can we quantify these factors to form a more accurate model for evaluating socially enhanced applications?" and is seen as a prime example of motivating research within this sphere. Quinn et al. (2011a, 2011b) analysed Facebook user profiles to monitor user activity levels and to investigate feature adoption, are examples of related research which encapsulates the overall issue that all users in a network are individuals. As motivation for our current studies we aim to assess the degree to which older users are currently engaging.

Assessing user behaviour within OSN data is complex in nature and highly problematic, primarily due to accessibility and privacy concerns of user data, with only a limited number of works in this discrete area. Subsequently, a lack of related datasets in related works is testament to these issues, whereby it is has been difficult to assess the individual interactions of users. As communication platforms they are multi-faceted with numerous features, the frequency and volume of communication across its numerous ties, means that interactions are exponentially greater than those of traditional, non-technology based social networks. Taking into account the wide variety of behaviours which may be exhibited and subsequently examined, a quantitative approach will be applied to areas considered to be of most relevance. Assessments will be made into both the engagement and activity of each cohort, quantifying any variances in behaviour. Issues concerning the connectedness and the adoption level of application features of each group; are viewed as central in determining and contrasting user behaviour(s). Interaction analysis of user interaction traces will present a micro level insight, assessing individual user behaviour(s) within these vast OSN applications. By understanding individual interactions we are then able to determine representative behaviours of related communities and cohorts.

In a series of five observational studies of user behaviour in Facebook, we review the current state of user interactions, quantifying the behaviour(s) of younger and older users. The precise behaviours were measured through the extraction and analysis of user generated content contained within the profile walls of each user. The studies make primary knowledge contributions in comparing the interactions of two disparate age cohorts based on their behaviours, which were all observed within one network. Results determined the degree to which variances exist between the following OSN components:

1 online friend numbers

2 period of engagement

3 application usage

4 engagement frequency

5 profile maintenance $(\mathrm{PM})$ rates. 
Through the analysis, we were able to answer the following five research questions, and provide insights into the impact of social interaction on younger and older users:

1 How many friends does the average user in each cohort contain?

2 How long have each cohort been actively engaged within their network?

3 How does application usage compare across each cohort?

4 Does the frequency at which users engage vary?

5 Does the rate at which each cohort maintains their profile vary?

The remainder of this paper is structured as follows. Section 2 discusses data collection, including issues of network selection and methodology. Section 3 details the result of data analysis for each of the studies. Section 4 provides a discussion on each result. A summary of the results is provided in Section 5 with their overall contributions assessed, with future work stated in conclusion.

\section{Data collection}

In the context of this paper, a 'younger user', refers to those who are between 15 and 30 years of age, whilst an 'older user' are those aged 50 years of age and over. As a point of note, the term older user is not to be confused with old age and the potential negative connation's it may hold, but is applied in a purely factual approach, whereby those over 50 are simply older than those 30 years and under. The rationale and methodology for network selection and the subsequent methods for data collection have been detailed below, along with definitions for all examined behaviours.

\subsection{Network selection}

"Millions of people use Facebook everyday to keep up with friends, upload an unlimited number of photos, share links and videos, and learn more about the people they meet." (Facebook, 2012)

A range of social networking services were evaluated for their potential use in the current study, based on a criteria of; data accessibility, popularity and user content. Given its overall impact, acceptance by users and the rich variety of behaviours that users can exhibit, Facebook was selected as the network on which to evaluate OSN behaviour. Official figures released in March 2012 reveal the number of monthly active users in Facebook reached 845 million (Facebook Statistics, 2012), an astonishing growth rate for a company established in February 2004. In relation to user functionality, the format of Facebook is to provide users with a 'wall' [Figure 1(a)], where users provide and control user generated content through updates on their status, post comments/replies, or share media content. Viewed in terms of either; revenue or user numbers, the format has proved extremely successful, with Facebook firmly established as the number OSN site (eBizMBA, 2012). 
Figure 1 (a) Facebook user wall (b) generated source code

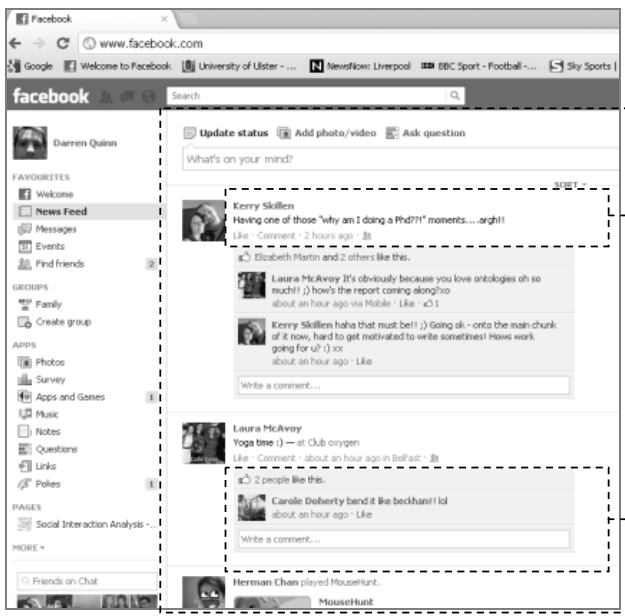

(a)

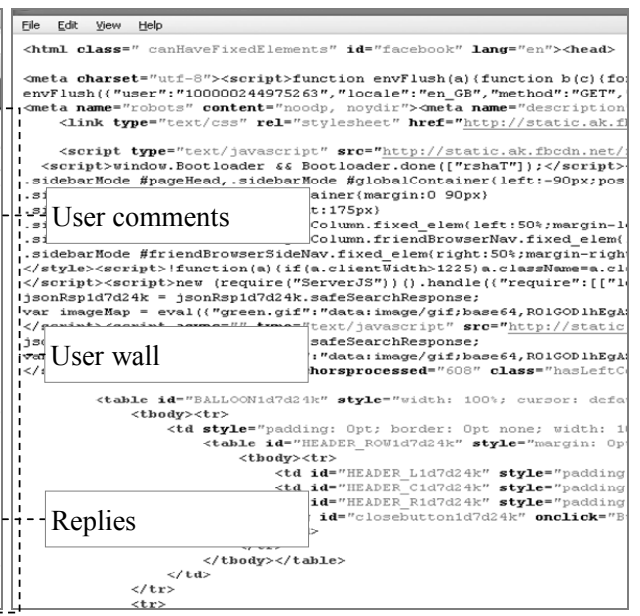

(b)

\subsection{Data gathering}

In order to examine user behaviours, the entire wall history and profile information of each user were acquired. Users were determined on two main criteria, these being that:

1 the full wall profiles of users were publically accessible

2 the age of the user was identifiable within their profile information.

As detailed below, the collection and comparison of user data involved a three stage process of; user evaluation, data extraction and data analysis. Profiles were obtained through the following method, altering only for user ages.

A Facebook user profile was created and using the 'find classmates' facility a list of potentially suitable 'friends' were identified and returned in a randomised order by Facebook. In a localised search, seeding points of the University of Ulster and Queens University Belfast were applied. Facebook enables users to access all older posts from a user's Wall when publically available. Each wall was exhausted until posts were unavailable, indicated by one of two stamp indicators:

1 'There are no more posts to show'.

2 '[Users First Name] joined Facebook'.

As age data is contained separately within the information page, it was acquired in conjunction with the generated source code of each user. Friends of each suitable user were then assessed for suitability; the process was then repeated until a sufficient amount of users were acquired. As users were sampled using a friend seed it is stated each profile was assessed strictly on their suitability for inclusion within each age cohort. The dataset was acquired in a two month period from December 2010, and analysed in a three stage process. 
1 Facebook walls containing user histories were manually extracted through the generated source code [Figure 1(b)].

2 Employing C\#.Net framework a bespoke parsing programme was developed for interaction analysis.

3 Comparison analysis was applied to address all issues of interest.

As stated, the approach of the study was to sample a localised population, whereby all users were considered to be retained within the UK. At the time of data collection in December 2010, Facebook's UK population was 28,940,400 (Clickymedia, 2010). The overall sample size volume of 500 user was determined based on a confidence level of $95 \%$ with a subsequent confidence interval of 4.5 from a population of $28,940,400$. As below, calculations determined the number of users required. Results revealed the sample size should be 474 . Where $\mathrm{z}=\mathrm{z}$ value (e.g., 1.96 for $95 \%$ confidence level), $\mathrm{p}=$ percentage picking a choice, expressed as decimal ( 0.5 used for sample size needed), $\mathrm{c}=$ confidence interval, expressed as decimal (e.g., $0.04= \pm 4$ ).

$$
\mathrm{SS}=\frac{\mathrm{z}^{2} *(\mathrm{p}) *(1-\mathrm{p})}{\mathrm{c}^{2}}
$$

\subsection{Dataset description}

In total 500 full user profiles were obtained (125 younger males, 125 younger females, 125 older males and 125 older females). The minimum age retrieved for younger users was 15 years with a maximum age value of 30 years, with a cohort mean of 21 years. In relation to older users; the minimum age retrieved was 50 years with a maximum age value of 83 , the cohort mean was 62 years.

\subsection{Definitions}

The following section defines terms that are referred to throughout the course of the study in relation to Facebook and the associated network structure.

- Public profile - privacy settings are set and controlled by each user, determining the accessibility of information. Users choose to disclose information to; 'everyone', 'friends of friends' or 'friends only'. Information disclosed to everyone is classed as 'public' and accessible by all registered Facebook users.

- Profile information - users are at liberty to provide character information in a range of areas. 'Basic information' elements consists of 'hometown', 'gender', 'date of birth', etc. 'education and work' information allows users to provide 'employer', 'university' and 'school' information including 'leaving year'.

- $\quad$ Profile age - all users do not explicitly state a date of birth, for those who did not a novel approach was applied. Profile attributes detail the university or school leaving year. If both or either were provided, it therefore became possible to estimate a user's age within a close proximity. 
- Active period - Facebook attaches a date-time stamp to each element, it becomes possible to determine an 'active period' for any given user, that being how long each user can be seen to have contributed to their network.

- $\quad$ PM rate- is the frequency at which a user amends their profile attributes, e.g., changing of profile picture or current location.

- Functional usage - interactions are defined as being any single measurable user activity. A comprehensive range of all potential activities were assessed, from which a list of six core activities were identified (Table 1). Activities were omitted if they were either a non-user activity (e.g., non-user comment/reply), or through the assessment of median values, whereby all low percentage values (assessed as $1 \%$ or less) were removed due to the insignificance of their overall functional impact.

Table 1 Core user activities

\begin{tabular}{l}
\hline Core activities \\
\hline User wall comment(s) \\
User reply(s) \\
User commenting on another user's wall \\
User commenting on another user's status \\
Use of a hyper media attachment \\
Use of a media application \\
\hline
\end{tabular}

Two specific examples of common occurrences within Facebook are provided to illustrate the defining and application of interactions and their subsequent analysis:

1 Use of a hyper media attachment (e.g., posting of a music video) is a single instance recorded as a user interaction and subsequently categorised within hyper media.

2 When a user posts a comment and another user posts a reply in response to this comment, both interactions are recorded. However, although two distinct interactions occur (one comment, one reply), only one is directly attributable to the user (i.e., the comment). The subsequent response is also recorded, but defined as a non-user interaction.

\section{Data analysis and discussion}

Results and discussion for each of the previously stated areas are outlined below. In relation to the discussion, following the presentation of results each aspect is examined, providing conclusions for each of the five research areas. Unless otherwise stated, it is to be assumed that in all studies, both cohorts contained the maximum number of users for each group.

\subsection{Friend numbers}

Analysis of friend values determined a number of key characteristics (Table 2). Fundamentally, analysis was aimed at establishing two aspects. Firstly, we were 
interested in acquiring the general population statistics concerning the friend number values. Secondly, as individual cohorts we needed to evaluate the distribution of user values, disclosing the overall group dynamics. Where appropriate; cohort values were examined in greater detail.

Table 2 Friend numbers statistics

\begin{tabular}{lcc}
\hline & Friend numbers \\
\hline & Older & Younger \\
\hline Mean & 85.02 & 482.87 \\
Standard error & 16.69 & 15.10 \\
Median & 37 & 428.5 \\
Standard deviation & 263.86 & 238.78 \\
Kurtosis & 160.06 & 1.45 \\
Skewness & 11.77 & 1.10 \\
Minimum & 0 & 84 \\
Maximum & 3,797 & 1,402 \\
Sum & 21,256 & 120,718 \\
Confidence level (95.0\%) & 32.87 & 29.74 \\
Confidence interval upper limit & 117.89 & 512.61 \\
Confidence interval lower limit & 52.15 & 453.13 \\
\hline
\end{tabular}

Analysis of results disclosed a significant variance between median and mean values in the case of older users. As detailed, the median value of 37, is less than half that of the mean score of 85. Detailed exploration disclosed the cohort contained a single outlying profile retaining 3,797 friends. Subsequently, it was revealed the profile in question was that of a public official who had adopted Facebook as a communication tool for constituents. As an individual profile, it artificially inflated the cohort mean. In order to assess its overall significance, the value was omitted and the cohort re-analysed. Resulting analysis revealed the median value remained at 37 . However, the cohort mean reduced by $17.65 \%$ to 70 .

Analysis of the statistics for both cohorts disclosed a clear correlation between age and friend numbers. Younger users are shown not only to contain a substantially higher mean friend value, but they also retain a broader distribution across a more expansive range. The median value of younger users returned an 11.6/1 ratio against older users. Significantly of the 250 older profiles, 129 were observed containing less than 40 friends, with $90 \%$ containing less than 140 friends. However, out of 250 younger users, 126 had less than 430 friends, with $90 \%$ having less than 830 friends. As illustrated in Figure 2, the distribution of values demonstrates the stark contrasts which can be observed between the cohort values. Measures of the population's skewness further disclosed that both cohorts follow an un-normalised distribution. However, although both cohorts contained a positive skew (i.e., the majority of values are left of the mean, with a longer tail to the right), the significantly high positive skew value relating to older users, emphasised the spread of friend values throughout the cohort. A further statistical measure of an unpaired $\mathrm{t}$-test further reaffirmed the statistical significance between the cohorts, returning a probability of less than 0.05 . It concluded the cohorts can be distinguished by a measure of friend values. 
Figure 2 Friend number distributions

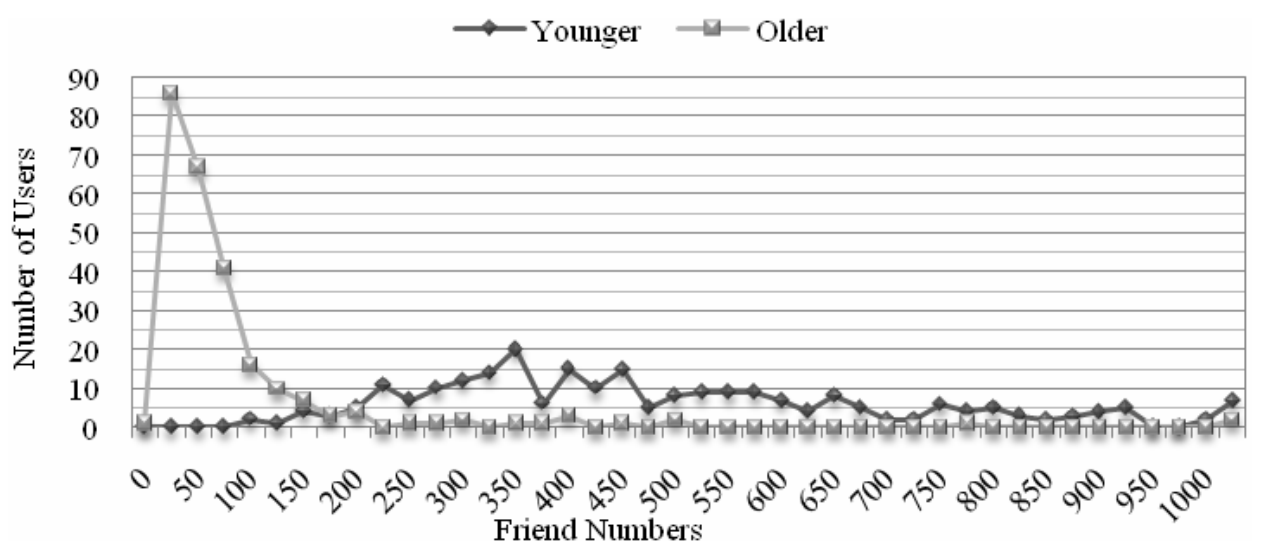

\subsubsection{Discussion}

The connectivity of each user and subsequently each cohort maybe determined through the assessment of user friend numbers. As the core demographic it was unsurprising that younger users contained a greater volume of friends compared to older users. However, the extent to which this was found to be true was still surprising. Results revealed that younger users have 11.6 times greater connections than that of older users. Although some research has discussed the issue of OSN 'friends' and the validity of categorising all connections as real 'friends' (Wilson et al., 2009), it is still possible from this study to draw conclusions.

As a social networking service, Facebook is designed specifically to increase opportunities for social engagement through a range of application functions. Regardless of whether each connection is a 'real' friend, what they can be seen to represent is an opportunity for social interaction. Connections made in a specifically social environment, such as Facebook have the potential to reduce the impact of social isolation. In this vain, older users were observed as having a significantly reduced amount of friend numbers. Analysis determined a correlation between friend numbers and age. Moreover, analysis highlighted the diversity in the ranges of friend values. Interpretations indicate that although smaller values are returned for older users, as a cohort they are connecting. As $90 \%$ of older users were shown to contain up to 140 friends, values allow for a conclusion to be drawn that connections are being established beyond that of immediate close friends and family. Analysis determines that although the number of connections is smaller in comparison to younger users, the older cohort is engaging and forming ties beyond their traditional real world social networks. Younger users were shown to have much more extended networks. As a cohort they typically interact frequently and form many connections. Friend values for younger users are significantly higher. However, given that the majority of younger users within the acquired dataset were from large institutions (i.e., college or university), interpretations of younger users having such large friend numbers are that connections are formed loosely, a view which is further supported given younger users increased real world social interactions. 


\subsection{Period of engagement}

Analysis of user activities derived a time frame known as the 'active period' for every user. Date-time stamps associated to each activity defined the length of time (in days) that each user can be observed as having contributed to their wall. As listed in Table 3, a range of measures have been applied to evaluate the variances between the two cohorts. Visualisation of the active period for each cohort is applied within 50 day ranges to directly contrast the active period for younger and older users.

Table 3 Periods of engagement

\begin{tabular}{lcc}
\hline \multicolumn{3}{c}{ Active period statistics (number of days) } \\
\hline & Older & Younger \\
\hline Mean & 208.80 & 428.08 \\
Standard error & 17.83 & 13.69 \\
Median & 74 & 384 \\
Standard deviation (STDEV) & 281.95 & 216.42 \\
Kurtosis & 2.33 & 4.28 \\
Skewness & 1.61 & 1.81 \\
Minimum & 1 & 1 \\
Maximum & 1,311 & 1,292 \\
Sum & 52,199 & 107,020 \\
Confidence level (95\%) & 35.12 & 26.96 \\
Confidence interval upper limit & 243.92 & 455.04 \\
Confidence interval lower limit & 173.68 & 401.12 \\
\hline
\end{tabular}

As illustrated within Figure 3, the periods to which the two cohorts have engaged can be seen to vary significantly. Statistical measures of the cohort's values enable a number of new observations to be drawn. Younger user's active period is significant, equating to 12.8 months, contrasting to that of older users who had a markedly lower median value equating to 2.5 months. Regarding younger users, analysis identified the principal range as being between that of 350 to 400 days, a range containing $27 \%$ of users. Older user's analysis disclosed 97 users were recorded as retaining 1 day of activity. Consequently, it is observed as the principal range, containing $39 \%$ of users. Results reveal that within the older cohort two categories of older users can be observed. Category A 'rejecters'; those who do not continue engaging beyond 1 day and do not return and; Category B 'maintainers' are those older users who maintain activity. Measures reported such as that of the STDEV expose the extent to which the populations within each cohort varied, with values of 216 days for younger users and 281 days for older users. As in the preceding study, an unpaired t-test confirmed that the results between the groups were significant, with a probability value less than 0.05 ; concluding the cohorts can be distinguished by a measure of their active period. 
Figure 3 Periods of engagement

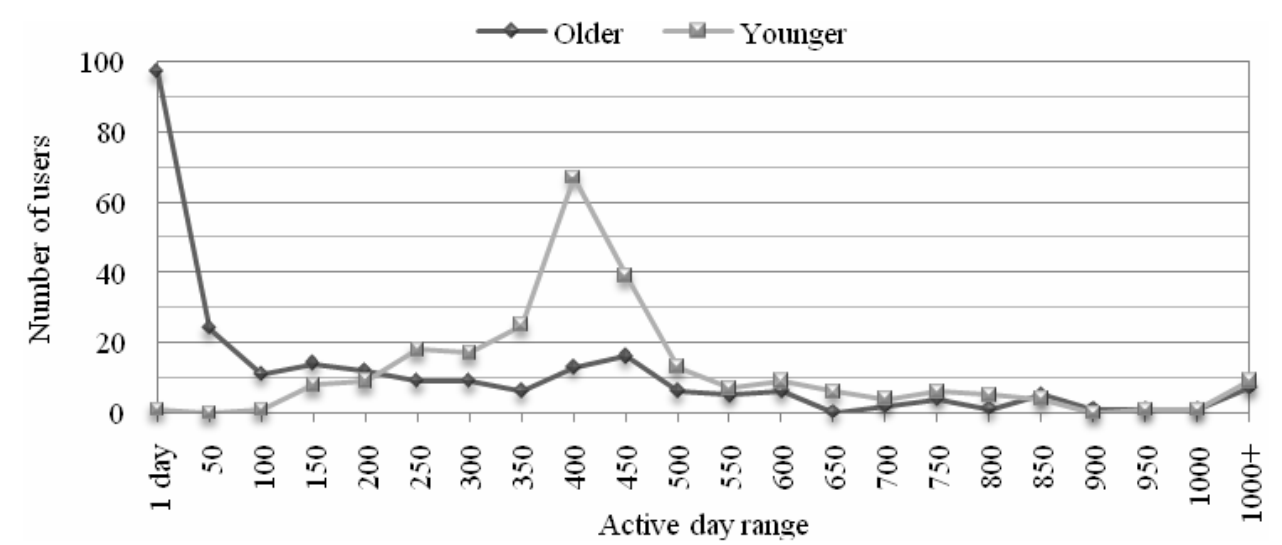

\subsubsection{Discussion}

As a discrete study, user walls were analysed to determine the number of days over which each user can be shown to have made a contribution. Notable variances between the cohorts were observed.

On average the profiles of younger users were shown to contain wall interactions for a period of 12.8 months, contrasted to 2.5 months for older users. However, of greater significance were the contrasts in users with an engagement period of 1 day. As a value it represented those users who created accounts and were active for a single day. In particular, a significant proportion of older users were associated with in this region (39\%). Interpretations are that two distinct categories are present; observed as containing rejecters and maintainers, i.e., those who create and maintain accounts and those who simply establish an account and make no further wall interactions. Distributions of those who do continue to engage indicate that it can be for a significant period of time. Conversely younger users contained just $1 \%$ within the one day range, with users being actively engaged over an extended period of time. Younger values revealed they are engaged to a greater degree. As 52\% were observed between 300 to 450 days, the cohort population is disclosed as largely containing established users. Generated content of younger users provided a rich volume of data. As a cohort their period of engagement was noted as being consistent and representative, whereby younger users are shown to be active engagers with their Facebook accounts. Both cohorts were seen to contain a broad range of users as witnessed through the significant standard deviation values and reinforced by their comparable maximum values. Results indicate a view that irrespective of volume; the longer the period of engagement, the higher the likelihood is that users will continue to employ Facebook as a modality for social interaction.

\subsection{Application usage}

As it would be unreasonable to assume that all users created accounts on the same day, or to have performed the same number of activities, analysing the functional usage over time was neither achievable nor realistic. However, comparisons of functional usage were able to be drawn from two perspectives. Firstly, analysis contrasted the total number of 
functions employed by every individual within each cohort. In doing so, it established the level of engagement for each cohort, determining a series of statistical measures, which were duly applied for comparative purposes (Table 4). Secondly; as user generated content disclosed every instance a function was applied, it therefore enabled an evaluation of the impact each function held for every individual's total usage. A uniform analysis required percentile values to be calculated, at individual and cohort level.

Within the context of this discrete study, only those functions directly attributable to the user were examined (i.e., non-user comments excluded). Following an evaluation on the impact of all functions, six functions were identified as being core across both cohorts, those being:

1 user comment

2 user reply

3 wall comment

4 status comment

5 hyper media

6 media application.

The results were as follows.

Table 4 Functional usage

\begin{tabular}{lcc}
\hline & Function usage statistics & \\
\hline Mean & Older & Younger \\
Standard error & 43.19 & 298.76 \\
Median & 6.62 & 18.77 \\
Mode & 5 & 203 \\
Standard deviation & 0 & 42 \\
Kurtosis & 104.68 & 296.71 \\
Skewness & 21.42 & 5.67 \\
Minimum & 4.24 & 2.00 \\
Maximum & 0 & 2 \\
Sum & 827 & 1,904 \\
Confidence level (95\%) & 10,798 & 74,690 \\
Confidence interval upper limit & 13.04 & 36.96 \\
Confidence interval lower limit & 56.23 & 335.72 \\
\hline
\end{tabular}

Analysis of cohort values disclosed a marked difference in mean values. Older users were observed as performing 43 activities, contrasted to that of 299 for younger users. As illustrated in Figure 4 the focus of younger users is upon providing comments and making status comments on the walls of other users. Combined, they accounted for $61.4 \%$ of all activity. As a subcategory, activities on other user profiles (i.e., providing wall and status comments) accounted for $25.4 \%$. Hyper media and media applications accounted for $20.1 \%$ of activity. It was observed that whilst $100 \%$ of younger user 
profiles contained activity, 60 older user profiles contained no activity (24\%). Of those profiles which contained activity, their focus is on providing user comments $(29.5 \%)$. Analysis disclosed younger users are more likely to provide comments (1.34/1) and 1.81 times more likely to post replies. Posting on other user's wall/status is comparable across the cohorts accounting for $25 \%$. However, older users post on the walls of other users at a rate of 2.66/1 to younger users, whereas younger users made a greater volume of status comments. A significant variance between the rate younger and older cohorts employed hyper media and media applications were observed, with $20 \%$ and $37 \%$, respectively. Although significantly lower in volume, normalised data observed older users as containing a broad spread of functionality usage. Younger users were shown to not only engage at a significantly higher volume, but their functional usage characteristics are diverse to those of older users. Results indicate a preference by younger users to update their personal status, whilst reflecting on updates posted by other users. Older users also make comments. However, as notable older characteristic they adopt hyper media and media applications to a greater extent.

Figure 4 Functional usage

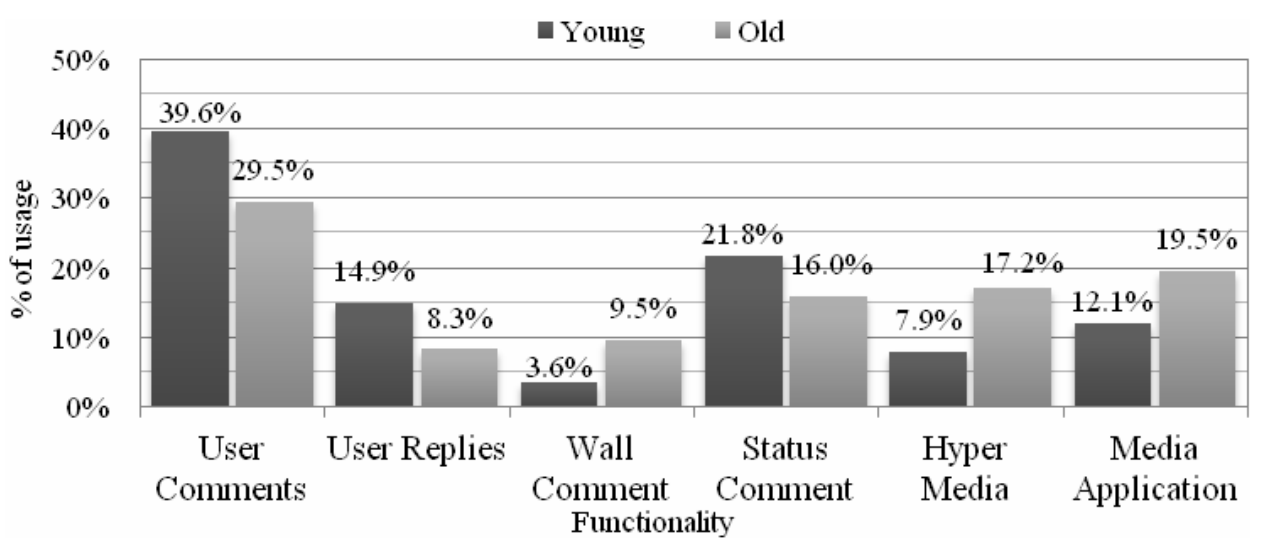

\subsubsection{Discussion}

Core application features were defined in relation to their impact upon usage for both cohorts, subsequently it allowed for an assessment of the activity level of both groups. Results determined the importance each function held.

Normalisations of user data and subsequent analysis revealed two distinct behavioural types. Older users were shown to have a broader spread of functionality usage in comparison to their younger counterparts and categorised as displaying the following characteristics. As a cohort they avail principally of user comments. However, other prominent behaviours are observed, particularly in the use of hyper media and media applications. Significant usage of both features illustrates they employ Facebook apps for gaming whilst also sharing links within their user walls to a greater degree than younger users. Interpretations of the behaviour by younger users are that although they share the same dominant feature of providing user comments, what is notable is the volume of comments made on the status of other users. As activities they are concerned with self-expression and reflection, as a cohort they express themselves more and are 
interested in the views of other users. An investigation of activity volume revealed the extent to which each cohort engage. The walls of younger users contained 299 activities, contrasted to that of 43 for older users. Results equated to younger users providing a mean of 115 user comments, compared to 14 for older users. Statistics disclose younger users as expressive, wanting to convey themselves and contribute to others who do so. Interpretations of statistics lead to conclusions that as a modality for social engagement, older users are more passive in their behaviour, whereby younger users are shown through their higher usage volumes to be instigators who will readily stimulate activity.

\subsection{Categorisation of user behaviour}

Analysis of user content derived a measure termed as 'activity frequency' for each individual. As detailed; date-time stamps provided a period into which the cumulative total of activities was divided. Resulting values were then applied as an overall frequency metric for the entire active period. Activity frequency calculation as below:

$$
\text { af }=n /(x 1-x 2)
$$

where activity frequency (af), first date stamp (x1), last date stamp (x2), total number of user activities (n).

Ascertaining an activity frequency metric for each individual enabled categorises to be established, which facilitated the contrasting of user behaviour across both cohorts. As listed in Table 5, five categories were developed, representing the activity range of users from ' $\mathrm{G} 1$ ' to 'G5'. In terms of analysis, user comment and reply frequencies were assessed and applied to contrast cohort behaviour.

Table 5 Activity frequency

\begin{tabular}{lcccccc}
\hline \multirow{2}{*}{ Category } & \multirow{2}{*}{ Number of days } & \multicolumn{2}{c}{ Comments } & & \multicolumn{2}{c}{ Replies } \\
\cline { 3 - 4 } \cline { 6 - 7 } \cline { 6 - 7 } G1 & & Younger & Older & & Younger & Older \\
\hline G2 & 0 & $1 \%$ & $52 \%$ & & $2 \%$ & $65 \%$ \\
G3 & $0-4$ days & $52 \%$ & $10 \%$ & & $24 \%$ & $2 \%$ \\
G4 & $5-9$ days & $23 \%$ & $6 \%$ & & $21 \%$ & $4 \%$ \\
G5 & $10-19$ days & $15 \%$ & $10 \%$ & & $21 \%$ & $4 \%$ \\
\hline
\end{tabular}

Analysis of comment frequencies for the younger cohort disclosed the majority of users engage frequently (within 4 days). The most prominent category being ' $\mathrm{G} 2$ ' accounting for $52 \%$ of all users. Notably; values were shown to decline throughout the remaining categories with; G3 containing 23\%, G4 15\% and G5 10\%. In relation to their reply frequencies, analysis disclosed a variance in comparison to comment behaviour. Category G5 contained the largest portion of younger users, accounting for $31 \%$. However, categories G2, G3 and G4 all returned similar values of $24 \%, 21 \%$ and $21 \%$ respectively. As with comments, category G1 was observed as accounting for a minority of users.

Analysis of older frequencies revealed that the majority of users do not engage. For those older users who do engage, they do so at a more extended rate. Specifically in 
relation to comments, the dominant category was G1, containing $52 \%$. As a category it identified users whose walls contained no activity. As detailed categories G2, G3 and G4 contained low values, ranging from $2 \%$ to $4 \%$. Reply categories for older users followed a similar trend to comments. $65 \%$ of older users were assigned to category G1. Category G5 recorded $24 \%$ of all user activity and was the most dominant category for older users in providing replies. Given an elevated G1 reply value (65\%) in comparison to a lower G1 comment value (52\%), it illustrates the heavier adoption of user comments by older users. In conclusion, results quantify the lesser degree to which older users engage, with younger users employing user comments on a more frequent basis.

\subsubsection{Discussion}

Categorising the rate at which users engage aimed to assess how both cohorts value Facebook. Distinct from other studies which assessed how often users logged onto their account (Benevenuto et al., 2009; Schneider et al., 2009; Viswanath et al., 2009), this study was designed specifically to measure how often a user made an active contribution. As a study, it provides a greater insight into user behaviour, determining how often each individual interacted. Findings were reflective of the preceding studies in that; older users were shown in both functions as largely being non-engagers, showing dominance in the category of NIL activity. Older users either; not engaging or at a significantly lesser frequency than their younger counterparts. Younger users were shown to provide user comments frequently, at a rate between 0-4 days. Analysis of younger reply frequencies determined significant values in each range as being inconclusive. However, given their natural life cycle, replies are anticipated as taking longer to generate and thus expected to occur less frequently. Conversely to the frequency of younger users, older users were noted for their absence of activity. Data analysis revealed the majority of older users failed to make contributions for either of the observed user comments or replies. Interpretations of both datasets are as follows; given their frequency of engagement, younger users are hypothesised as attaching a higher significance to the use of Facebook for social interactions. Older users on the contrary were observed as engaging in the higher ranges (i.e., at a slower rate), with interactions occurring primarily over 20 days. Low frequency engagement depicts a subsequent view that for the majority of older users Facebook is at present, not a primary mode for social interaction. Analysis enables conclusions to be drawn that for younger users, communicating through this modality is a common occurrence. One which witnesses a high volume of activity as observed in the preceding study. It would be misleading to assume that a frequent interaction rate is to be naturally associated with a higher value being associated to the use of Facebook by either group. However, what may be concluded from the results is that given their frequency of engagement, it can now be stated that more so for younger users, Facebook is important for their interactions, accounting for a significant proportion of their communications.

\subsection{Profile maintenance}

Facebook profiles provide a host of information on each individual. Within Facebook users maintain their profile as often or as little as they choose. As listed in Table 6, 13 profile options were fully available to all users at the time of data capture. 
Table 6 PM activities

\begin{tabular}{lccc}
\hline No & Attribute & No & Attribute \\
\hline 1 & Profile picture & 7 & Looking for \\
2 & Work info & 8 & Activities \\
3 & Quotations & 9 & Gender \\
4 & About me & 10 & Languages \\
5 & Current location & 11 & Schools and universities \\
6 & Hometown & 12 & Interested in \\
& & 13 & Interests \\
\hline
\end{tabular}

Analysing the rate at which individuals maintained their profile established a measure of importance for online identity within each group. For all users, the total number of PM activities were acquired, and subsequently divided into the total number of active days, a metric determining the mean value for any individual. Resulting analysis established a series of further descriptive population statistics, applied to contrast PM activity, as listed within Table 7. The results of PM frequency analysis were as follows.

Table $7 \quad$ PM statistics

\begin{tabular}{lcc}
\hline & All users PM statistics & \\
\hline & Younger & Older \\
\hline Mean & 40.02 & 56.89 \\
Standard error & 3.57 & 7.81 \\
Median & 24.96 & 4.04 \\
Mode & 0.00 & 0.00 \\
Standard deviation (STDEV) & 56.44 & 123.56 \\
Sample variance & $3,185.25$ & $15,266.03$ \\
Kurtosis & 55.32 & 27.28 \\
Skewness & 6.06 & 4.53 \\
Minimum & 0 & 0 \\
Maximum & 646 & 1,042 \\
Sum & $10,005.80$ & $14,221.60$ \\
Confidence level (95\%) & 7.03 & 15.39 \\
Confidence interval upper limit & 47.05 & 72.28 \\
Confidence interval lower limit & 32.99 & 41.50 \\
\hline
\end{tabular}

As illustrated in Figure 5, users from both cohorts were allocated within five day ranges. Those users with no PM activity were recorded as 'NIL'. Analysis disclosed stark variances in the PM rates of both cohorts. Older users were observed as strongly dominating the most frequent range of ' $0-5$ ' days, whilst younger users peaked in the 15-20 day range, declining thereafter. Results observe that $75 \%$ of all younger users have a PM rate of less than 45 days, with 'NIL' values accounting for $1.6 \%$. The $100+$ day range accounted for $7 \%$ of all younger users, an expansive range between $100+$ days to 646 days. Analysis of older users stated 35\% of all changes occurred between 0-5 days (88 users). As a cohort, $75 \%$ of older users maintain their profiles below 70 days, 
although the number of NIL users recorded totalled 38 , accounting for $15 \%$ of the group. Significantly the $100+$ day range accounted for $18 \%$ of all older users, a range for users who maintained their profile between 100 to 1,042 days.

Figure 5 PM rates

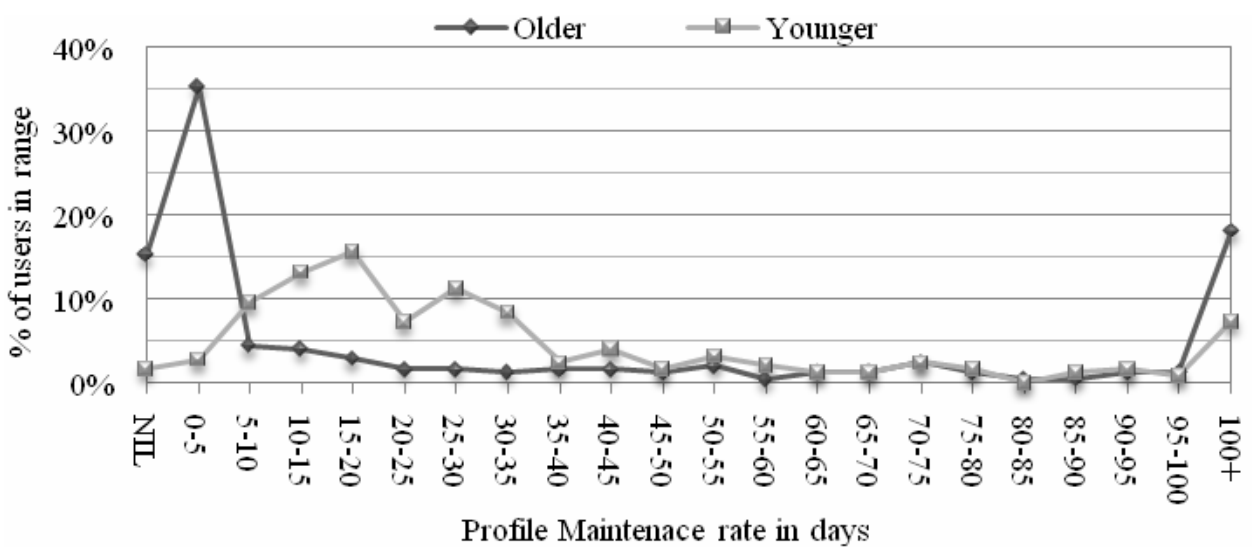

Although results indicate a more frequent rate by older users, results are somewhat contradictory given the larger volume of older user NIL values. Furthermore, older users are observed as containing a greater 100+ day range compared to younger users, with maximum values of 1,042 days and 646 days respectively. Analysis disclosed both cohorts follow an un-normalised distribution with both cohorts observed as comprising a positive skew. The imbalance within the older cohort is further reflected within recorded STDEV values.

\subsubsection{Discussion}

In assessing the rate at which users maintain their profiles, the overarching aim was to determine the significance each cohort attaches to their online profiles. The rate provides a valuable understanding concerning the importance online identity and promotion of self holds for both groups.

Results initially indicated older users performed PM activities between 0-5 days, a frequency much higher than that of younger users who were shown to maintain their profiles between 15-20 days. However, contradictions were noted whereby $33 \%$ of users, contained either a value of; 'NIL' or ' $100+$ ' days. Inconsistency in older user users results were further compounded with a markedly larger max PM value of 1,042 days, compared to 646 days. Analysis disclosed that irregularities were born as a result of those older users who had an active day range of just 1 day. Users with just 1 day were noted as performing a 'PM' activity. Subsequently, due to the large contingent of older users falling into this category it artificially raised the rate at which older users maintain their profile, a result which is misleading given that this cohort of user do not continue to engage. The distortion of older values means that direct comparisons are unable to be drawn for the entire dataset. However, given that the vast majority of older users within the $0-5$ are those with an active day of value of 1 , for analysis purposes this range can largely be excluded. Remaining data indicates that older users either; do not maintain 
their profiles, or for those that do, engage at long intervals, a view reinforced by values contained within the ' $100+$ ' day ranges. Results imply that maintaining profiles is carried out with no degree of frequency or consistency for older users. In light of the consistent active period for younger users, analysis of values enables a more reliable view of the PM rates. In general, younger users were noted as maintaining within 44 days, with $75 \%$ of users captured. Results conclude activity is being carried out with a degree of consistency across the cohort.

Overall consideration is to be given to the dynamics of each cohort; for younger users aspects measured such as that of: education or employer, are characteristics which will be still quite fluid. Attributes which will evolve over the course of this age period. Older users as a cohort are established, the maintenance of their online characteristics being at low frequency is viewed as being entirely consistent with the cohort dynamics.

\section{Summary}

Results of an exploration into the interaction analysis of user interaction data form a new body of knowledge, extending the current understanding into how younger and older users compare in their interactions and behaviour within Facebook. As a cohort, the mid age range (between 31 to 49 years) was intentionally omitted for the following reasoning. The study aimed to determine the engagement levels of older OSN users, investigating OSN as a potential technology to alleviate the impact of increasing social isolation brought about through old age. As a group social isolation is not deemed as critically important for the mid-range as it is for today's older users. As such, their evaluation is viewed as being outside the scope of current studies. Subsequently, five discrete studies analysed and contrasted user behaviours. The aim of the studies was to assess how older users engage in comparison to the core demographic of younger Facebook users. Wall profiles containing the generated content of users were extracted and analysed, determining metrics at both an individual and cohort level. Analysis of user data focused on determining knowledge within five areas. As discrete issues they were selected to enhance the understanding into the fundamental aspects involving OSN engagement. Results of cohort comparisons have provided the following awareness.

In summary, the variances of older user engagement in Facebook have been quantified against younger users. Older users are engaging and forming networks, however, not to the extent of younger users. The degree to which older users fail to return and maintain activity is significant; of those who do, they are seen to maintain activity. Reasoning as to why older users fail to return can only be speculated and determined through a qualitative approach. Functional differences determined a different behavioural type to that of younger users, engaging widely with the available functions. In conclusion, differences in behaviour are that, older users engage with less frequency and with a significantly lower volume of interactions. Their employment of applications functions is observed as being characteristically different to younger users. However, it appears that as an approach to counter act social isolation there may be merits in its use to keep older users connected. In the case of both cohorts, the volume of interactions cannot be taken as a measure of worth the service holds for each user, i.e., a low volume older user, may well attach an equal worth to that of a high volume younger user. As a specific area of interest, the relationship between usage and impact is to be assessed. 
In future work, it is hoped that this current body of work can be extended to contain a review of all ages through a large-scale data collection approach if the significant challenges of privacy and security can be overcome. Future works in this area aim to provide a more in-depth analysis through increased data mining techniques to uncover greater insights. However, direct engagement with network operators for collaborative research appears as the most likely long-term option for successful future endeavours. As a multi-disciplinary area it is viewed that greater insights may also be gained through joined up research into user behaviours within a computer science context. Work is currently ongoing in the assessment of user interactions and their perceived impact upon quality of life/wellbeing through a qualitative assessment of both cohorts.

\section{References}

Akcora, C., Carminati, B. and Ferrari, E. (2011) 'Network and profile based measures for user similarities on social networks', Proceeding of IEEE International Conference on Information Reuse and Integration, pp.292-298.

Ala-Mutka, K.D., Broster, R., Cachia, C., Centeno, C., Feijóo, A., Haché, S., Kluzer, S., Lindmark, W., Lusoli, G., Misuraca, C., Pascu, Y., Punie, Y. and Valverde, J.A. (2009) 'The impact of social computing on the EU Information Society and Economys', JRC Scientific and Technical Report, EUR 24063 [online] http://ipts.jrc.ec.europa.eu/publications/pub.cfm?id=2819 (accessed 2 April 2011).

$\mathrm{Au}$ Yeung, C. and Iwata, T. (2011) 'Strength of social influence in trust networks in product review sites', in Proceedings of the Fourth ACM International Conference on Web Search and Data Mining (WSDM '11), ACM, New York, NY, USA, pp.495-504.

Benevenuto, F., Rodrigues, T., Cha, M. and Almeida, V. (2009) 'Characterizing user behavior in online social networks', Proceedings of the 9th ACM SIGCOMM Conference on Internet Measurement Conference (IMC '09), ACM, New York, NY, USA, pp.49-62.

Bennett, S., Maton, K. and Kervin, L. (2008) 'The 'digital natives' debate: a critical review of evidence', British Journal of Educational Technology, Vol. 39, No. 5, pp.775-86.

Burke, M., Kraut, R. and Marlow, C. (2011) 'Social capital on Facebook: differentiating uses and users', Proceedings of ACM CHI 2011: Conference on Human Factors in Computing Systems, pp.571-580

Chau, D., Pandit, S., Wang, S. and Faloutsos, C. (2007) 'Parallel crawling for online social networks', Proceedings of the 16th International Conference on World Wide Web, ACM, pp.1283-1284.

Chen, K., Zhou, Y., Song, L. and Yang, X. (2011) 'Building artificial identities in social network using semantic information', Proceedings of International Conference on Advances in Social Networks Analysis and Mining (ASONAM), pp.565-566.

Cheung, C.M.K., Chiu, P. and Lee, M.K.O. (2011) 'Online social networks: why do students use Facebook?', Computers in Human Behavior, Vol. 27, No. 4, pp.1337-1343.

Chew, H.E., LaRose, R., Steinfield, C. and Velasquez, A. (2011) 'The use of online social networking by rural youth and its effects on community attachment', Information, Communication \& Society, Vol. 14, No. 5, pp.726-747.

Clickymedia (2010) 'Facebook User Statistics January 2010' [online] http://www.clicky.co.uk/2010/01/facebook-user-statistics-january-2010/ (accessed 31 July 2012).

Corbett, P. (2010) 'Facebook Demographics and Statistics Report 2010', 1 April [Online] http://www.istrategylabs.com/2010/01/facebook-demographics-and-statistics-report-12010145-growth-in-1-year (accessed 12 April 2012). 
Coyle, C.L. and Vaughn, H. (2008) 'Social networking: communication revolution or evolution?', Bell Labs Technical Journal, Vol. 13, No. 2, pp.13-17.

eBizMBA (2012) 'Top 15 most popular social networking sites' [online] http://www.ebizmba.com/articles/social-networking-websites (accessed 12 April 2012).

Facebook (2012) [online] http: http://www.facebook.com/facebook?v=info (accessed 12 April 2012).

Facebook Statistics (2012) [online] http://newsroom.fb.com/content/default.aspx?NewsAreaId=22 (accessed 12 April 2012).

Ferrara, E. (2012) 'Community structure discovery in Facebook', Int. J. Social Network Mining, Vol. 1, No. 1, pp.67-90.

Gomes, A.K. and Pimentel, M. (2011) 'Social interactions representation as users behavioral contingencies and evaluation in social networks', Proceedings of Fifth IEEE International Conference on Semantic Computing (ICSC), pp.275-278.

Greene, J., Choudhry, N., Kilabuk, E. and Shrank, W. (2011) 'Online social networking by patients with diabetes: a qualitative evaluation of communication with Facebook', Journal of General Internal Medicine, Vol. 26, No. 3, pp.287-292.

Grenade, L. and Boldy, D. (2008) 'Social isolation and loneliness among older people: issues and future challenges in community and residential settings', Australian Health Review, Vol. 32, No. 3, pp.468-478.

Hanson, V.L. (2009) 'Age and web access: the next generation', Proceedings of the 2009 International Cross-Disciplinary Workshop on Web Accessibility, W4A, Madrid, Spain, ACM, New York, NY, pp.7-15.

Hossmann, T., Legendre, F., Nomikos, G. and Spyropoulos, T. (2011) 'Stumbl: using Facebook to collect rich datasets for opportunistic networking research', Proceedings of IEEE International Symposium on a World of Wireless, Mobile and Multimedia Networks, pp.1-6.

Howe, N. and Strauss, W. (2000) Millennials Rising: The Next Great Generation, Vintage, New York.

Krasnova, H. and Veltri, N.F. (2010) 'Privacy calculus on social networking sites: Explorative evidence from Germany and USA', Proceedings from the 43rd Hawaii International Conference on System Sciences, Honolulu, HI, USA, pp.1-10.

Kwai, F.I.P.R. and Wagner, C. (2008) 'Weblogging: a study of social computing and its impact on organizations', Decision Support Systems, Vol. 45, No. 2, pp.242-250.

Lenhart, A., Madden, M. and Hitlin, P. (2005) 'Teens and technology: youth are leading the transition to a fully wired and mobile nation', Pew Internet \& American Life Project, Washington DC [online] http://pewinternet.org (accessed 12 April 2012).

Lindley, S.E., Harper, R. and Sellen, A. (2009) 'Desiring to be in touch in a changing communications landscape: attitudes of older adults', Proceedings of the 27th International Conference on Human Factors in Computing Systems, Boston, MA, USA, pp.1693-1702.

Mislove, A., Marcon, M., Gummadi, K., Druschel, P. and Bhattacharjee, B. (2007) 'Measurement and analysis of online social networks', Proceedings of the 7th International ACM SIGCOMM Conference on Internet Measurement, ACM, pp.29-42.

Newman, M. and Leicht, E. (2007) 'Mixture models and exploratory analysis in networks', Proceedings of the National Academy of Sciences, Vol. 104, No. 23, pp.9564-9569.

Parameswaran, M. and Whinston, A.B. (2007) 'Research issues in social computing', Journal of the Association for Information Systems, Vol. 8, No. 6, pp.336-350.

Prenksy, M. (2001) 'Digital natives, digital immigrants', On the Horizon, Vol. 9, No. 5, pp.1-6.

Quinn, D., Chen, L. and Mulvenna, M. (2011a) 'An examination of the behaviour of young and older users of Facebook', Proceedings of the International ICST Conference on eHealth 2011, pp.9-16. 
Quinn, D., Chen, L. and Mulvenna, M. (2011b) 'Does age make a difference in the behaviour of online social network users?', Proceedings of the 2011 International Conference on Internet of Things and 4th International Conference on Cyber, Physical, and Social Computing, pp.266-272.

Sayago, S. and Blat, J. (2009) 'About the relevance of accessibility barriers in the everyday interactions of older people with the web', Proceedings of the 2009 International Cross-Disciplinary Conference on Web Accessibililty (W4A) (W4A '09), ACM, New York, NY, USA, pp.104-113.

Schneider, F., Feldmann, A., Krishnamurthy, B. and Willinger, W. (2009) 'Understanding online social network usage from a network perspective', Proceedings of the 9th ACM SIGCOMM conference on Internet measurement conference (IMC '09), ACM, New York, NY, USA, pp.35-48.

Tapscott, D. (1998) Growing Up Digital: The Rise of the Net Generation, McGraw-Hill, New York.

Ur, B.E. and Ganapthy, V. (2009) 'Evaluating attack amplification in online social networks', Web 2.0 Security and Privacy.

Victor, C., Scambler, S. and Bond, J. (2009) The Social World of Older People. Understanding Loneliness and Social Isolation in Later Life, McGraw Hill, Berkshire.

Victor, C.A., Scambler, S.J., Bowling, A. and Bond, J. (2005) 'The prevalence of, and risk factors for, loneliness in later life: a survey of older people in Great Britain', Ageing \& Society, Vol. 25, pp.357-375.

Viswanath, B., Mislove, A., Cha, M. and Gummadi, K.P. (2009) 'On the evolution of user interaction in Facebook', Proceedings of the 2nd ACM Workshop on Online Social Networks (WOSN '09), ACM, New York, NY, USA, pp.37-42.

Wilson, C., Boe, B., Sala, A., Puttaswamy, K.P. and Zhao, B.Y. (2009) 'User interactions in social networks and their implications', Proceedings of EuroSys '09: The 4th ACM European Conference on Computer Systems, pp.205-218.

Wilson, S.M. and Peterson, L.C. (2002) 'The anthropology of online communities', Annual Review of Anthropology, Vol. 31, pp.449-467.

Xiang, J.N. and Rogati, M. (2009) 'Modeling relationship strength in online social networks', in Workshop on Analyzing Networks and Learning with Graphs, December.

Ying, X. and Wu, X. (2009) 'On link privacy in randomizing social networks', $P A K D D$. 